\title{
HIGH-FREQUENCY 3D GEOMORPHIC OBSERVATION USING HOURLY TERRESTRIAL LASER SCANNING DATA OF A SANDY BEACH
}

\author{
K. Anders ${ }^{1,2}{ }^{*}$, R. C. Lindenbergh ${ }^{3}$, S. E. Vos ${ }^{4}$, H. Mara ${ }^{2}$, S. de Vries ${ }^{4}$, B. Höfle ${ }^{1,2,5}$ \\ ${ }^{1}$ 3D Geospatial Data Processing Research Group (3DGeo), Institute of Geography, Heidelberg University, Germany - (katharina.an- \\ ders, hoefle)@uni-heidelberg.de \\ ${ }^{2}$ Interdisciplinary Center for Scientific Computing (IWR), Heidelberg University, Germany - hubert.mara@iwr.uni-heidelberg.de \\ ${ }^{3}$ Department of Geoscience \& Remote Sensing, Delft University of Technology, The Netherlands - r.c.lindenbergh@tudelft.nl \\ ${ }^{4}$ Department of Hydraulic Engineering, Delft University of Technology, The Netherlands - (s.e.vos, sierd.devries)@tudelft.nl \\ ${ }^{5}$ Heidelberg Center for the Environment, Heidelberg University, Germany
}

Commission II, WG II/10

KEY WORDS: 4D geospatial data, change analysis, 3D time series, multitemporal point clouds, LiDAR, beach monitoring

\begin{abstract}
:
Geomorphic processes occur spatially variable and at varying magnitudes, frequencies and velocities, which poses a great challenge to current methods of topographic change analysis. For the quantification of surface change, permanent terrestrial laser scanning (TLS) can generate time series of 3D point clouds at high temporal and spatial resolution. We investigate how the temporal interval influences volume change observed on a sandy beach regarding the temporal detail of the change process and the total volume budget, on which accretion and erosion counteract. We use an hourly time series of TLS point clouds acquired over six weeks in Kijkduin, the Netherlands. A raster-based approach of elevation differencing provides the volume change over time per square meter. We compare the hourly analysis to results of a three- and six-week observation period. For the larger period, a volume increase of $0.3 \mathrm{~m}^{3} / \mathrm{m}^{2}$ is missed on a forming sand bar before it disappears, which corresponds to half its volume. Generally, a strong relationship is shown between observation interval and observed volume change. An increase from weekly to daily observations leads to a five times larger volume change quantified in total. Another important finding is a temporally variable measurement uncertainty in the 3D time series, which follows the daily course of air temperature. Further experiments are required to fully understand the effect of atmospheric conditions on high-frequency TLS acquisition in beach environments. Continued research of 4D geospatial analysis methods will enable automatic identification of dynamic change and improve the understanding of geomorphic processes.
\end{abstract}

\section{INTRODUCTION}

Geomorphic processes shape natural landscapes in any topographic setting at different magnitudes, frequencies, and velocities. A fundamental part of improving our understanding of these spatially variable processes is their observation and quantification of properties, such as location, size, shape, deformation, and movement rate. For recording the topography of an area at (sub-)cm-accuracy, terrestrial laser scanning (TLS) is a technique capable of acquiring billions of 3D point measurements of surfaces in a target scene using the Light Detection and Ranging (LiDAR) principle, and obtaining a 3D point cloud of the scene. Through repeated scanning, the time dimension is added to the point cloud data and the $4 \mathrm{D}$ geospatial dataset serves as basis to quantify change over time (Eitel et al., 2016). The analysis is ideally conducted at an interval appropriate to the change rate of the observed phenomenon. This can be highly variable for natural landscapes from slow-rate movement, such as the creeping of a rock glacier (e.g., Avian et al., 2009), to rapid displacements, such as flowing lava (e.g., Crown et al., 2013).

To obtain LiDAR data at high temporal resolution of daily to subhourly intervals over longer periods, permanent TLS is an emerging acquisition technique (Eitel et al., 2016). In this static setup, the instrument records a target scene from a fixed position in regular intervals typically defined by the operator for the intended application. To date, permanent TLS has been deployed in few use cases, such as landslides (Kromer et al., 2017; Höfle et al.,
2016), rockfall monitoring (Williams et al., 2018), and sandy beaches (Vos et al., 2017). In this research, we use the 3D time series of a beach in the Netherlands acquired by Vos et al. (2017).

Sandy beaches are a topographic setting characterized by highly complex behavior of wind- and wave-driven sand transport occurring at multiple temporal and spatial scales (Cowell et al., 2003). The combination of these multiscale sand transport processes, for example, contributes to the restoration of the beach after a storm event. Particularly the corresponding dune-ward transport of sand is not well understood. High-frequency 3D time series data enable to analyze sand transport at high temporal detail and with the geometric measurement accuracy of TLS data.

In this paper, we investigate how an hourly TLS time series improves morphologic process observation for a sandy beach. We hypothesize that change processes can be observed at greater temporal detail with the hourly data. Typically, multitemporal data is acquired at intervals of months to years, such as the countrywide annual airborne LiDAR surveys conducted in the Netherlands (AHN, 2018). For such low acquisition frequencies, the multitemporal data can contain the states before and after the evolution or deformation of a morphologic form. Then it is not possible to derive the process characteristics, such as movement rate or a gradual change in shape. Further, at higher temporal resolution the time series can disclose change that is completely missed

* Corresponding author 
at lower observation intervals. For example, erosion and accretion have a counteracting effect on the volume budget if they successively occur at the same location on the beach in between repeated acquisitions.

Further, there is an opportunity to use data sampled at high temporal resolution to reduce the uncertainty of measurements and improve the level of detection of an observation. With level of detection, we refer to the minimum detectable change between time steps. This is influenced by different factors:

- The instrument's measurement accuracy

- The scanning geometry, i.e. measurement range and incidence angle at each location within the target scene

- The alignment accuracy of the multitemporal point clouds

- Physical properties of the recorded target, such as surface roughness and laser return intensity (LRI)

- Temporally variable atmospheric conditions, such as temperature, humidity, and air pressure

With high-frequency sampling, errors in elevation measurement can be identified by comparing to temporal neighbors, i.e. the same location measured at previous and successive points in time (Kromer et al., 2015). This increases the confidence of quantified surface change and can hence improve the level of detection. In the following, we outline related research on TLS time series used for geomorphic change observation.

An hourly time series of point clouds was used by Williams et al. (2018) to assess the magnitude-frequency distribution of rockfalls on a cliff. The authors specifically address how the acquisition interval influences the derived event frequency of rockfalls. As rockfalls often coincide or coalesce, multiple discrete events can only be identified individually at an adequate acquisition interval.

Comparing to the observation of sand transport on the beach, there is an important conceptual distinction in the type of change process that is observed. Rockfalls occur as single, irreversible events with exclusively eroding effect on the topography, meaning that there is no process opposing the loss of volume. Thus, for the case of continuous observation, it would be possible to distinguish every single rockfall event. Surface change on the beach takes place as (potentially) continuous process of sand being transported away from and to a location depending on the conditions of wind direction and velocity, wave forcing, as well as external influences such as intentional shifting of sand. This adds a new aspect of relevance to high-frequency sampling and change analysis for observation of geomorphic processes.

Continuous surface change is studied by Kromer et al. (2017) for a landslide. Using a half-hourly TLS time series made it possible to identify deformation preceding the failure of the slope into a rockfall event. The observed deformation occurred over a period of six days, so that it would likely not have been captured through conventional multitemporal TLS surveying. For their TLS time series, the authors describe a noticeable effect of temporally variable environmental conditions on data quality. For example, point density and the total number of measurements were strongly reduced for acquisitions during and after rainfall events, as the reflectivity of targets was lowered due to wetness of the surfaces. Permanent laser scanning implies data acquisition through varying atmospheric conditions over time, which influences the repeated LiDAR measurements. The influence of atmospheric conditions on TLS range distances is known (e.g. Hejbudzka et al., 2010), but difficult to assess as it is not practicable to measure all relevant variables directly on site (Fey and
Wichmann, 2017). Further, the properties of the recorded target and the scanning geometry influence the accuracy of the recorded TLS data (Soudarissanane et al., 2011).

Different methods have been established to account for these accuracy issues in multitemporal point clouds. Systematic errors that occur, for example, due to movement of the sensor between acquisitions, might require an alignment correction, which is typically done based on stable areas in the target scene (Wujanz et al., 2018). All residual distances of stable parts between multitemporal point clouds determine the level of detection for change quantification. To reduce this uncertainty in $3 \mathrm{D}$ time series, Kromer et al. (2015) propose temporal averaging, which makes use of point redundancy in the temporal domain within local neighborhoods. This approach requires the averaging window to be much smaller than the observed change rate to avoid averaging out actual change and consequently lose information. Thus, data acquisition at very high frequency increases the ability to improve the level of detection without smoothing the temporal trend of the observed process itself.

Against this background, we examine the 4D dataset of the beach for the level of detection and temporally variable measurement uncertainty associated to the $3 \mathrm{D}$ point clouds in the time series. The primary objective is to analyze the data for change processes under particular consideration of the influence of the observation frequency on the quantified volume budget. This shows the benefit of high-frequency sampling for the aspect of reversible changes that are completely lost to the observer depending on the temporal resolution of available data.

We investigate how the temporal observation interval influences the quantified volume change regarding (i) the temporal detail of the change process and (ii) the volume budget regarding missing change information due to the counteracting effects of accretion and erosion. For this, we quantify the volume change on the beach for every time step of the time series and assess how the result is influenced by temporal resolution for exemplary change occurrences on the beach: overall accretion and erosion through sand transport and the higher-magnitude formation of a sand bar. With this, we contribute first steps of a new analysis approach for 3D time series acquired by permanent TLS for the use case of a sandy beach. We further strengthen the concept of 4D geomorphic observation enabled by temporally high-resolution TLS.

\section{DATA AND METHODS}

In this section, we present the data and explain the methods used for analysis, for which the main steps are depicted in Fig. 1.

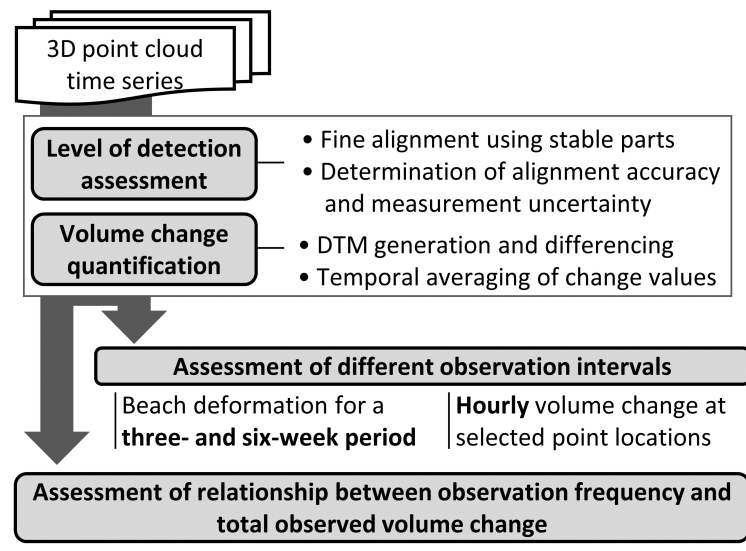

Figure 1. Overview of main analysis steps 


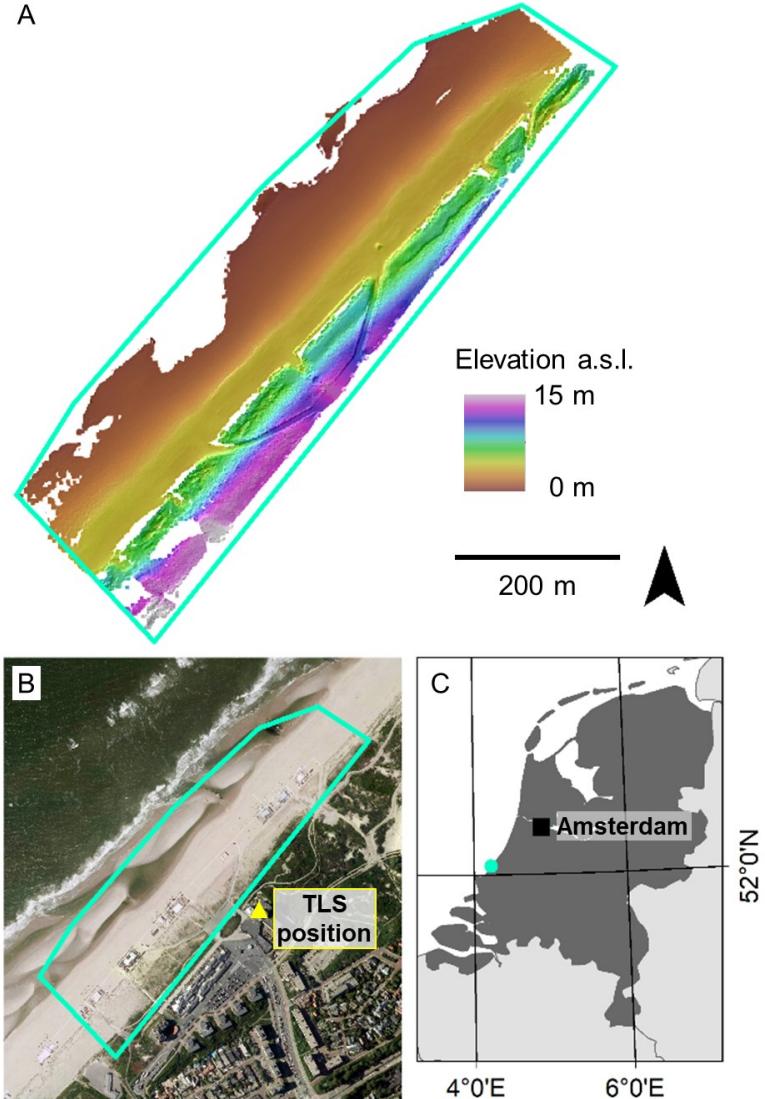

Figure 2. (A) Terrain elevation of the study site (based on TLS data) with extent designated in (B) aerial imagery of the beach at Kijkduin. (C) Overview map with location of the study site in the Netherlands. Data: Aerial imagery (C) pdok.nl 2017, World Borders (C) thematicmapping.org 2017

\subsection{Study Site and Dataset}

The 3D time series dataset used in this paper is acquired on the beach of Kijkduin in the Netherlands (52 04 '14" N 4 ${ }^{\circ} 13$ '10" E, Fig. 2). A Riegl VZ-2000 TLS (Riegl LMS, 2017) was mounted in a stable reference frame on a hotel roof overlooking the beach in the winter season of 2016/2017 (cf. Vos et al., 2017). In this paper, we use the hourly $3 \mathrm{D}$ point cloud dataset of the period from 2017-01-15 to 2017-02-28. We choose this subset of the time series as it follows a storm event in the days before and contains particular change occurrences associated to beach restoration. The time series subset acquired at the study site is complete except for a few missing timestamps due to poor meteorological conditions (cf. Section 2.2). Data was acquired every full hour with a vertical and horizontal point spacing of $9 \mathrm{~mm}$ at $10 \mathrm{~m}$ measurement range. Each day at noon, an additional high-resolution scan was acquired with a point spacing of $2 \mathrm{~mm}$ at $10 \mathrm{~m}$ measurement range. The target area of the beach is at a range of approximately $100-600 \mathrm{~m}$ from the sensor on the hotel roof. For every scheduled scan, latest weather information was retrieved online and passed to the TLS for temperature, air pressure, and humidity correction of the LiDAR measurements.

All point clouds in the time series were fine aligned to the highresolution scan of the first day (2017-01-15). The rigid transformation matrix of each point cloud to the global reference was computed for stable parts distributed in the scene (pavement, façades, information signs; Fig. 3), using an Iterative Closest Point method (Besl and McKay, 1992).
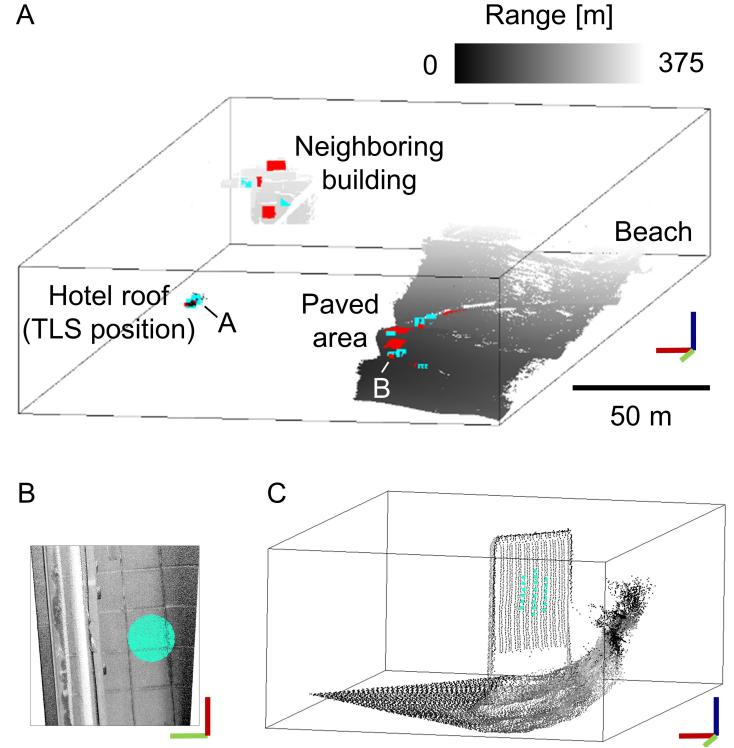

Figure 3. (A) Extract of the point cloud scene colored by range with stable parts used for fine alignment (red) and control planes (blue). Below, exemplary control planes are shown

(B) on the tiled floor near the scanner and (C) on an information sign next to the dunes

Measurements with a Global Navigation Satellite System receiver were acquired in Real-Time Kinematic mode for georeferencing of the TLS data using one fixed global transformation matrix.

\subsection{Level of Detection Assessment}

2.2.1 Accuracy of Multitemporal Point Cloud Alignment We determine the alignment accuracy between multitemporal point clouds based on planar surfaces that are expected to be stable throughout the acquisition period, but independent from the parts used for fine alignment. We choose ten control planes on the pavement, building façades and information signs with different orientation distributed in the stable area adjacent to the beach area itself (Fig. 3).

\begin{tabular}{|c|c|c|c|c|c|c|}
\hline ID & $\begin{array}{c}\text { Loca- } \\
\text { tion }\end{array}$ & $\begin{array}{c}\text { Range } \\
{[\mathrm{m}]}\end{array}$ & $\begin{array}{c}\text { Incidence } \\
\text { Angle }\left[{ }^{\circ}\right]\end{array}$ & $\begin{array}{c}\text { LRI } \\
{[\mathrm{dB}]}\end{array}$ & $\begin{array}{c}\text { Point count } \\
\text { (ref. / comp.) }\end{array}$ & Surface type \\
\hline 1 & $\mathrm{P}$ & 158 & 80 & -10 & $34 / 3$ & Pavement \\
2 & $\mathrm{P}$ & 106 & 77 & -11 & $123 / 8$ & Pavement \\
3 & $\mathrm{P}$ & 85 & 72 & -9 & $252 / 17$ & Pavement \\
4 & $\mathrm{P}$ & 89 & 72 & -10 & $227 / 13$ & Pavement \\
5 & $\mathrm{P}$ & 141 & 60 & -5 & $191 / 11$ & Info sign \\
6 & $\mathrm{P}$ & 87 & 70 & -7 & $387 / 22$ & Info sign \\
7 & $\mathrm{H}$ & 3 & 62 & -8 & $350 \mathrm{k} />23 \mathrm{k}$ & Balustrade \\
8 & $\mathrm{~N}$ & 333 & 2 & -4 & $69 / 5$ & Façade \\
9 & $\mathrm{~N}$ & 360 & 2 & -5 & $56 / 5$ & Façade \\
10 & $\mathrm{H}$ & 5 & 75 & -11 & $>60 \mathrm{k} />3 \mathrm{k}$ & Tiled floor \\
\hline
\end{tabular}

Table 1. Location and properties of control planes in the paved area $(\mathrm{P})$, on the neighboring building $(\mathrm{N})$, and on the hotel roof $(\mathrm{H})$. Given properties are the measurement range to the TLS sensor, incidence angle, laser return intensity (LRI) and number of points representing the plane in the reference (highresolution) and an exemplary compare point cloud

The properties of the control plane locations are listed in Tab. 1. The given incidence angle of the LiDAR measurement is defined as the angle between the laser beam originating from the TLS sensor and the local surface normal at the measurement location 
(Soudarissanane et al., 2011). In the flat terrain of the beach environment, we assume all surface normals to be oriented vertically, i.e. calculated the incidence angle to the perfectly horizontal plane. Only for the control planes in vertical position, the incidence angle is calculated using the plane surface normal.

To determine the alignment of two point clouds, we fit planes to the global reference point cloud at the location of the control planes within fixed 3D search radii of $0.3 \mathrm{~m}$. Subsequently, we compute the mean point-wise distances of $3 \mathrm{D}$ points in the compare point cloud to the reference planes. For each point cloud in the time series, we average the mean distance values obtained for all control planes. The final average value describes the alignment accuracy of two point clouds.

\subsubsection{Temporally Variable Measurement Uncertainty}

In addition to the overall alignment of multitemporal point clouds, we examine the temporal variability of measurements at individual locations. The measurement error in the point clouds can vary over time, for example, due to changing atmospheric conditions, and cause measurement uncertainty, which propagates into the quantification of surface change. As the distance to the sensor is constant over time for stable surfaces, any deviation of repeated elevation measurements at these locations, beyond random noise, indicates errors.

We derive measurement variability as the difference in elevation to a fixed reference, according to the raster-based method described in Section 2.3.1. We choose this simplified method of one elevation value per pixel location in the raster having confirmed a high correspondence to the distribution of originally measured $\mathrm{Z}$ coordinates in the $3 \mathrm{D}$ point cloud data at these locations.

We expect the degree of this variability to be dependent on the scanning geometry. Given the setup of the permanent laser scanner and the topographic setting, it is not possible to separate the variables range and incidence angle for this dataset, as both increase more or less linearly towards the beach (cf. Fig. 2). Therefore, we consider two locations at different distances from the TLS sensor (cf. Tab. 2). Location A is situated on the pavement in the paved area close to the dunes but not on the sandy area itself. Location B is situated on the upper part of the beach, which is generally subject to sand transport but assumed to be quite stable on the short-term during calm periods.

\begin{tabular}{|c|c|c|c|c|}
\hline ID & Location & $\begin{array}{c}\text { Range } \\
{[\mathrm{m}]}\end{array}$ & $\begin{array}{c}\text { Incidence } \\
\text { Angle }\left[^{\circ}\right]\end{array}$ & Surface type \\
\hline A & Paved area & 106.6 & 76.5 & Pavement \\
B & Beach & 148.5 & 76.9 & Sand \\
\hline
\end{tabular}

Table 2. Locations and respective properties used to assess temporally variable measurement uncertainty

To assess a potential link to atmospheric conditions, we use meteorological data from the same period and check the values for similar variability compared to elevation measurements at the point locations. As there is no meteorological station at the study site, we use data from Hoek of Holland (approximately $12 \mathrm{~km}$ southwest of Kijkduin), namely hourly measurements of air temperature, precipitation, wind speed, and wind direction (KNMI, 2017). Further, we use tide height data from Scheveningen (approximately $6 \mathrm{~km}$ northeast of Kijkduin) (Rijkswaterstaat, 2017).

\subsection{Volume Change Quantification}

\subsubsection{DTM Generation and Differencing}

We choose a raster-based approach for quantifying the volume change in the time series dataset, given the flat topography and low microtopographic variability of the study site. Thus, the volume change at any location on the beach is given by the elevation change resulting from the difference between two terrain elevation rasters over the area of a pixel. These Digital Terrain Models (DTMs) are derived from each 3D point cloud in two steps. First, we filter off-terrain points based on the relative height of points over the local minimum in a neighborhood of $1.0 \mathrm{~m}$. We set the filter threshold to $0.2 \mathrm{~m}$ to account for surface roughness and the slightly sloping terrain morphology. Then we rasterize the DTM using the median elevation value within a cell size of $1.0 \mathrm{~m}$. We fill gaps in the DTMs with the mean value in the one pixel neighborhood of empty pixels. We use the software OPALS (Pfeifer et al., 2014) to generate the DTMs.

By stacking all volume change rasters of the time series, we obtain a 3D matrix of volume change per pixel area $(2 \mathrm{D}+$ time $)$. Each $2 \mathrm{D}$ coordinate in the matrix represents a location on the beach and volume change over time at this location can be extracted as 1D time series using the respective pixel values along the temporal dimension.

\subsubsection{Temporal Neighborhood-Based Averaging}

The 1D time series of volume change at a location can contain values that notably deviate from their temporal neighbors. These can represent either sudden change within the continuous change process at a location or outliers caused by other effects. To identify exceptional change values in the time series, we use the temporal neighborhood for applying the median filtering approach of Eltner et al. (2017). The method is based on the 4D filtering approach proposed by Kromer et al. (2015) which filters noise both in the spatial and temporal dimensions. We do not adopt the spatial filtering for our dataset as the terrain is already filtered in the frame of the DTM generation (Section 2.3.1).

The averaging is applied using a moving median, i.e. by setting the change value at a point in time to the median of all values within a temporal window around the point. We use a temporal window of 48 hours. Thus, all values 24 hours prior and successively are considered. We keep the raw change values for analysis and use the averaged time series in the presentation of results.

\subsection{Influence of Temporal Resolution on the Observation}

The derived time series of volume change over time provides the basis to investigate the influence of temporal resolution on the observation. We first analyze elevation change on the beach for two dates in our time series compared to the reference date, 2017-02-06 and 2017-02-28, corresponding to a three-week and six-week period, respectively. This serves to assess the change that is derived from data acquired at lower temporal resolution similar to a conventional multitemporal LiDAR survey. From the volume change on the beach resulting for these periods, we select exemplary locations that are examined at higher temporal detail. Based on this, we assess the gained detail on the underlying morphologic change process.

Subsequently, we investigate the implication of hourly sampling on the observed sand transport. We quantify the total observed volume change in a defined sub-area $(90 \times 130 \mathrm{~m})$ for every possible observation frequency in the time series, i.e. at hourly intervals up to a six-week interval. Via this relation, we can derive how much information is missed as change is being eliminated 
between acquisitions due to the accretion and erosion counteracting on the volume budget.

\section{RESULTS AND DISCUSSION}

\subsection{Level of Detection}

\subsubsection{Accuracy of Multitemporal Point Cloud Alignment} Resulting from the plane-based assessment (Section 2.2.1), the full period of the time series used in this paper achieves a mean alignment accuracy of $0.4 \mathrm{~cm}$ with a standard deviation of $0.2 \mathrm{~cm}$. Fig. 4A contains the result plotted for a subset of the time series. The plane-based alignment has distinct outliers for some time stamps and there is a temporal variability of values.

To examine a possible dependence of high values on the properties of individual control planes, we show the mean point distances over time per control plane in Fig. 4B. For better visability, we separate the plots into two groups of control planes. Group 1 has generally low point-to-plane distance values. These planes are located on the hotel roof, with a very consistent result, and on the neighboring building as well as in the paved area, with more variable values, assumingly due to the higher measurement range (plane IDs 1-4 and 7-10, cf. Tab. 1). The large peak on 2017-01-24 derives from a series of point clouds acquired during a heavy rain event. These points in time appear as temporal gaps in the analysis, as they do not contain data in the target area of the beach due to strongly decreased measurement ranges under the conditions of heavy rain.

Group 2 in Fig. 4B illustrates two control planes in the scene that have a much larger variability of point-to-plane distances and thus strongly influence the final, averaged alignment accuracy value per point cloud (Fig. 4A). These control planes are located on information signs in the paved area before the beach (plane IDs 5-6, cf. Tab. 1).

\section{A}

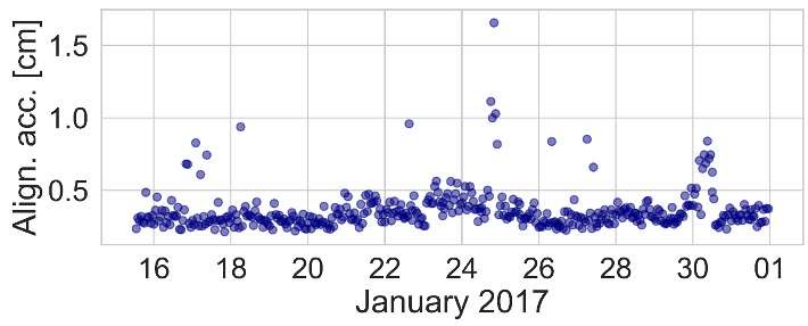

B

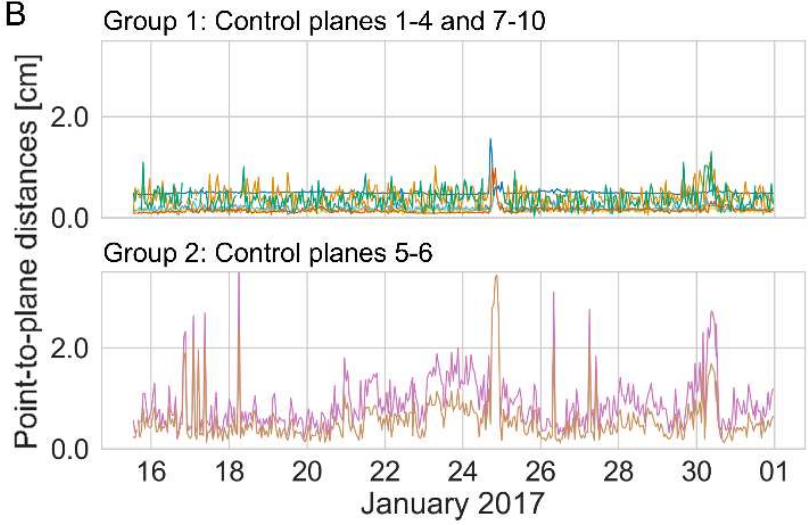

Figure 4. A) Alignment accuracy of hourly scans to the reference point cloud. B) Mean absolute point-to-plane distances per control plane (distinguished by color) separated into two groups with (1) little variability, and (2) strong fluctuation of values

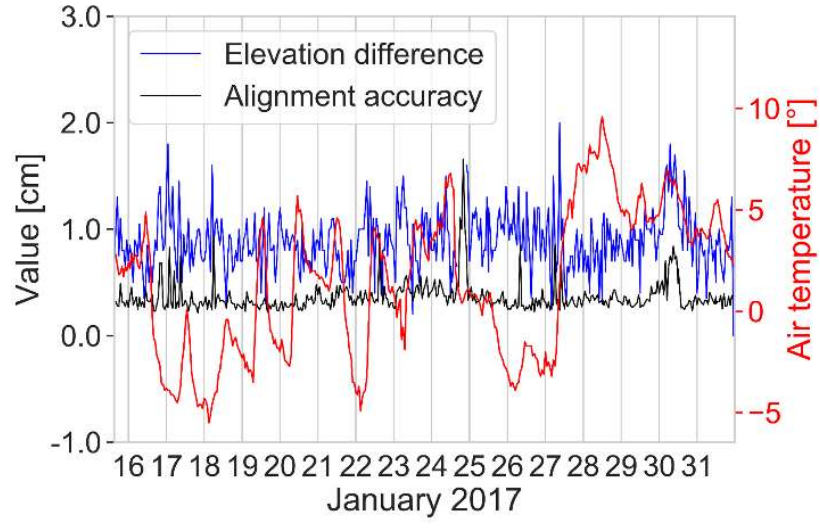

Figure 5. Relation of elevation differences to the course of air temperature. Elevation difference is quantified using the DTM differences at a location on the beach that is assumed to be subject to little change throughout the displayed period

\subsubsection{Temporally Variable Measurement Uncertainty}

We compare deviations in elevation measurements at stable locations to the course of meteorological and tide height data (Section 2.2.2). We did not find a relation with any of the variables, except a visual correspondence to the course of air temperature. The plot in Fig. 5 depicts air temperature compared to elevation differences for the location on the beach, expected to be stable throughout the period of the assessment (Tab. 2, location B).

The course of measurement deviation on the beach follows the course of air temperature, intermittently in an inverse manner (Fig. 5). For the location on the pavement (Tab. 2, location A), the fluctuation of measurement deviations is strongly visible only before the fine alignment of point clouds, but with lower magnitude than on the beach. One possible explanation is an influence of air temperature on the material of the TLS mounting frame, which leads to slight tilting of the sensor over time. As the area of the beach is at larger distance to the sensor, deviations due to angular misalignment of scans increases towards the beach, which is lacking any stable parts, though, and cannot be included in the alignment correction. Considering the pattern of measurement deviation, we hypothesize that there is an effect in the LiDAR measurement itself through factors such as humidity or near surface moisture, which are indirectly linked to air temperature and influence the refraction of the laser beam. The component of this effect which is linear in range is removed by the point cloud alignment.

Regarding the presented temporally variable measurement deviation, the minimum detectable change in this study cannot be expressed primarily with the obtained alignment accuracy values as commonly expected from TLS data. A possible solution in future permanent TLS setups on the beach could be to place targets for fine alignment in the area itself or at further distance beyond. The installation of fixed targets in the sandy ground that are stable over long periods and under the variable external forcing of wind and waves will pose a great challenge. We do not investigate further improvements of the data here and accept that the level of detection is hence strongly limited in the target area on the beach, as the focus of our paper is on the change analysis of the $3 \mathrm{D}$ time series.

\subsection{Volume Change Quantification}

We analyze geomorphic change over time on the beach based on the quantified volume change (Section 2.3.1). Fig. 6 contains the result for the two observation periods of three and six weeks. 


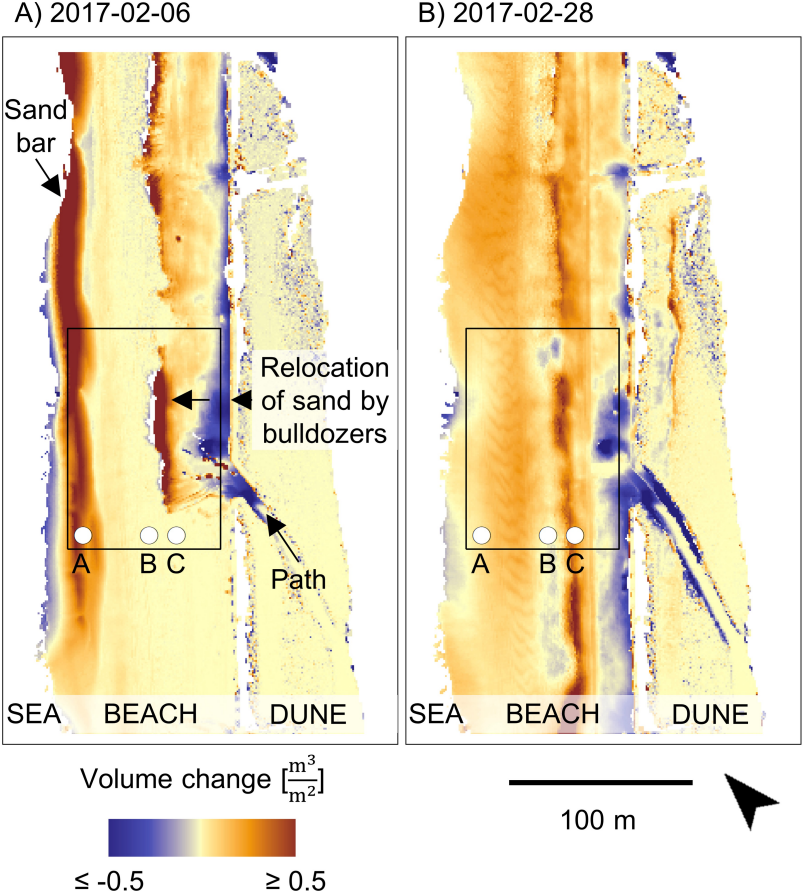

Compared to: 2017-01-15

Figure 6. Volume change for a period of (A) three weeks and (B) six weeks as DTM difference to the reference. Points A-C mark the locations used for the time series assessment (Section 3.3). The black rectangle marks the area used to assess the volume budget at different observation intervals (Section 3.3.3)

The volume change over the three-week period (Fig. 6A) shows little change over most of the beach area, but high volume increase and decrease for local areas. Near the path from the paved area to the beach, an area of large volume decrease is visible.

This is the result of bulldozer works after the storm event immediately before the onset of our time series, to which we compare the topographic state three weeks later. These works relocated the sand towards a lower part on the beach, appearing as elongated accumulation form spread out in front of the higher beach part. Towards the sea, a very distinct accretion form along most of the beach stretch is visible. This form represents a sand bar, which developed after the storm from sand being transported landwards by waves.

From the volume change over the six-week period (Fig. 6B), it becomes visible that more bulldozer works of relocating sand took place and large volumes of sand are distributed along the center of the entire beach. The sand bar, which was distinctly visible three weeks before, has disappeared. The comparison to the previous date in Fig. 6A suggests that the sand forming the sand bar is now spread out almost evenly over the beach area, which is indicated by the overall, small volume increase dune-wards from the (former) sand bar.

The results for the three- and six-week period exhibit how certain change processes are lost to the analysis at larger acquisition intervals. If only the result of the six-week period were available, the formation and disappearing of the sand bar would be completely missing. Further, it does not necessarily become apparent from the displayed results, what change processes the different structures of volume change represent and what caused their formation. A temporal resolution of three weeks already provides a valuable 3D point cloud time series with much higher return intervals than commonly available, for example with annual countrywide surveys (cf. Section 1). Still, the analysis is still lacking important parts of information. With higher-frequency sampling, the formation of the sand bar can be characterized in temporal detail. Sudden changes, such as the relocation of sand through human interference, can be located in the temporal domain and analyzed for its effects over time. This aspect is subject of the next section.

\subsection{Influence of Temporal Resolution on the Observation}

In this section, we pick three exemplary locations for which we extract the volume change on the beach as $1 \mathrm{D}$ time series. The locations are marked in Fig. 6: A) a sand bar location, B) a location on the lower part of the beach, and C) a location on the border between the upper and lower part that is subject to relocation of sand by bulldozers. Fig. 7 displays the time series of these locations as both raw and temporally averaged change values.

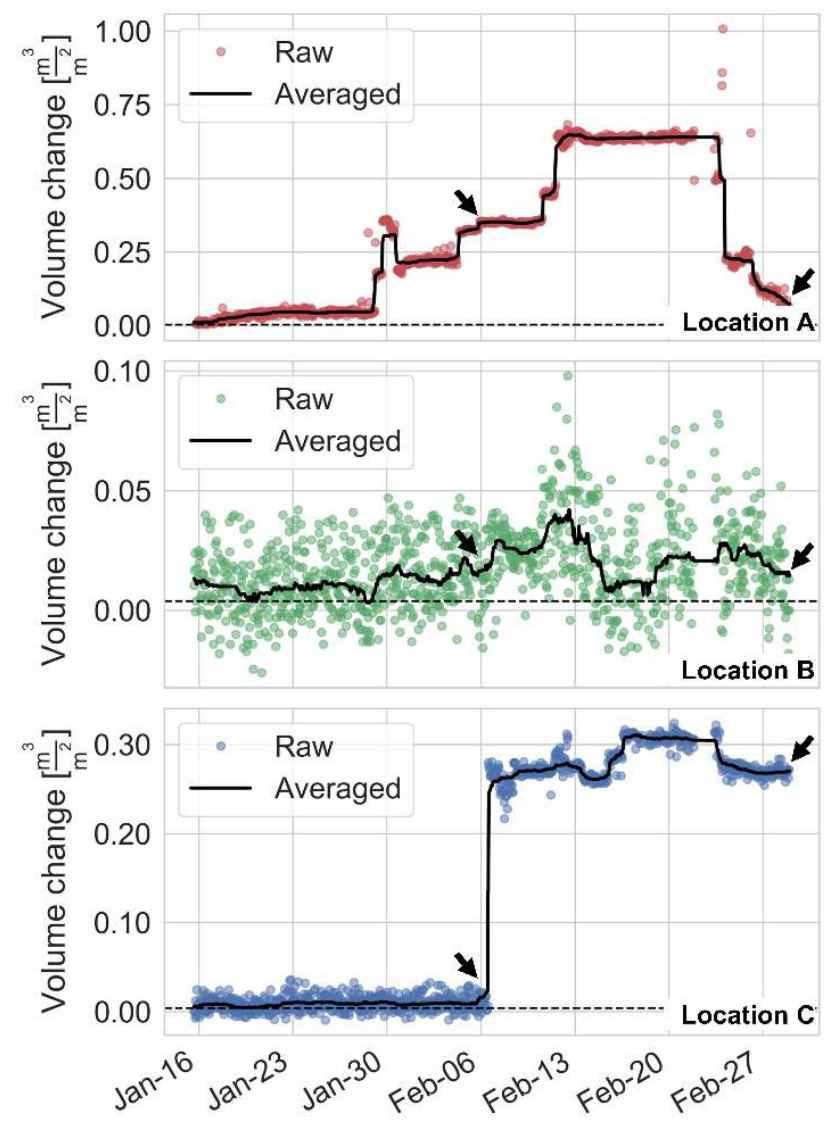

Figure 7. Time series of volume change at locations A-C (Fig. 6). Black arrows mark the points in time of the three- and six-week period. Raw values are visualized together with the result of the temporal averaging. The dashed line marks the alignment accuracy derived for the entire time series. Vertical axes have different scales

\subsubsection{Temporal Neighborhood-Based Averaging}

The averaged line of the temporal median in Fig. 7 visualizes that the volume change values are smoothed to the longer-term change. The raw values are more scattered, which could be caused both by the temporal measurement deviation (Section 3.1), or represent temporary change as actual volume change. Thus, for change analyses at hourly to daily scales, the uncertainty given by the level of detection takes a large influence. 
A better distinction of these two aspects will require to improve the level of detection. This can be achieved through advanced 4D filtering by considering the spatial domain. For this, we could leverage spatial autocorrelation inherent in morphologic change processes. If similar change values occur in the neighborhood of a location, it is more likely they represent actual surface change.

\subsubsection{Observation of Change Process Details}

The hourly time series of locations A-C in Fig. 7 enable a description of the occurring change processes at high temporal detail. For comparison to results of the three- and six-week period, respectively, black arrows in the time series mark the points in time which are displayed in Fig. 6.

Location A is situated on the sand bar (Fig. 6A). With the higherresolution time series, it can now be traced how the sand bar developed at this location and a more or less gradual accretion over time becomes visible. Compared to the three-week period of the volume change raster, the time series also discloses that the sand bar had a state of much higher volume at this location, which remained over a period of approximately two weeks. Thus, for a three-week interval, a volume increase of $0.3 \mathrm{~m}^{3} / \mathrm{m}^{2}$ is missed, which corresponds to almost half the volume of the sand bar at this location. While the sand bar has formed gradually, it disappears quickly starting on 2017-02-24 during a storm and strong rainfall event. Though point cloud data is missing at the exact time due to these meteorological conditions, the point in time can be determined from the averaged time series within a time frame of few hours. Location B exhibits only small-scale change values fluctuating between volume increase and decrease. The result might be caused at least to some degree by the temporal effects in the LiDAR measurements and represent the corresponding fluctuation of the level of detection (cf. Section 3.1). The course of change values at Location $\mathrm{C}$ is disrupted by the accumulation of sand through the bulldozer work. This can be seen in the time series in the sudden volume increase on 2017-02-06. The volume increase occurs immediately after the data presented in Fig. 6A was acquired. This emphasizes that the three-week analysis only contains a short snapshot of the morphologic state of the beach, which only little time later is heavily reworked.

The time series at the selected locations reveal that a higher temporal resolution has a strong influence on the detail of beach observation and ultimately on the information that is extracted in the geomorphic analysis and interpretation. Only through the dense time series in the period of sand bar formation and disappearance a detailed insight can be gained on the geomorphologic process, rather than only the identification of its existence. The next step of the geomorphic object observation will be to extract its spatial properties and ultimately derive the spatial-temporal characteristics, such as change in shape and movement rate.

\subsubsection{Relation of Observation Interval and Observed Vol- ume Budget}

In the previous section, we present examples of change processes on the beach, where geomorphic observation can strongly benefit from higher-frequency data acquisition of at least daily intervals. Besides the gain in temporal detail on the geomorphic process, the sand bar example illustrates that for larger return periods important increases in volume are lost, as they are eliminated before the next data acquisition.

Fig. 8 visualizes the relation of observation interval and observed volume change. We perform this assessment for the area marked by the black rectangle in Fig. 6 and quantify the average total volume change per square meter.

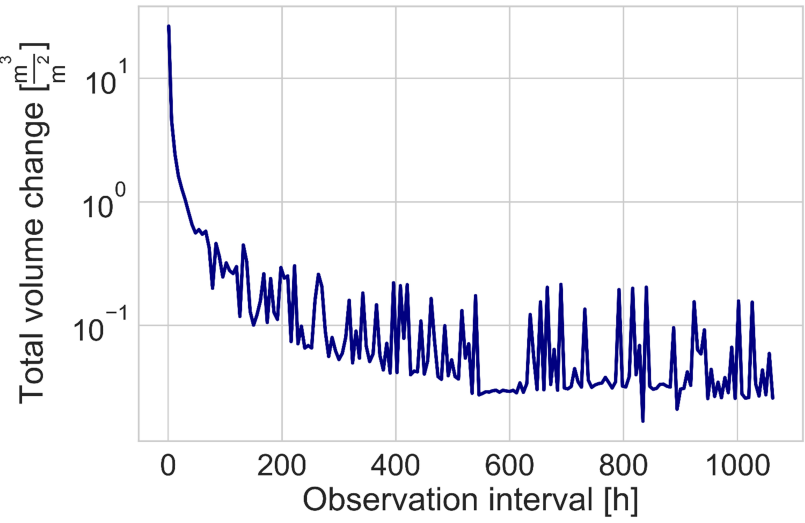

Figure 8. Relation of observation interval to the volume budget observed in the time series dataset over a period of six weeks

The relation exhibits that there is a large influence of the temporal resolution on the observed volume budget. The total volume change starts to increase particularly strongly when the interval is decreased to less than 100 hours, i.e. approximately four days. The larger the interval of the analysis, the more occurrences of accretion and erosion are lost to the observation. In our study over a period of six weeks, with a decrease of the observation interval from daily to weekly, the amount of sand that is missed in the analysis corresponds to around 10-14 truckloads of sand being transported within an area of $10 \times 10 \mathrm{~m}^{2}$. Further, the variability of observed change for increasing intervals emphasizes how the timing of acquisition greatly influences the observation.

It needs to be noted that the measurement error contained in each of the datasets accumulates in the quantified volume. To evaluate the observed volume budget including small-scale changes at high temporal resolution, the error contribution requires quantification. In the future, this can be investigated with independent measurements or using an experimental setup with an additional TLS instrument simultaneously recording a target area from a different perspective and at a closer measurement range.

\section{CONCLUSIONS AND OUTLOOK}

In this paper, we investigate the analysis of a TLS time series of high temporal resolution for the observation of surface change on sandy beaches. These change processes are dominated by highly dynamic sand transport leading to both accretion and erosion in local areas of the beach.

Our change analysis shows that the high temporal resolution of the $3 \mathrm{D}$ time series provides a source of $4 \mathrm{D}$ geoinformation for an improved understanding of morphologic change processes on the beach. By increasing the temporal interval of the analysis over long periods, object information and process characteristics can be extracted at more appropriate temporal levels of detail than in conventional TLS surveys, with either high-frequency acquisition over short periods or large return intervals.

Further factors need to be investigated to better assess the spatially and temporally variable uncertainty of change analysis, particularly regarding the measurement effect in the high-frequency TLS data. From our results, this seems to be related to atmospheric conditions, influencing the LiDAR range measurement to deviate in inverse correspondence to the course of air temperature. Future permanent laser scanning setups on the beach should examine this more closely by performing complementary measurements of on-site atmospheric conditions, such as temperature 
and humidity. Moreover, the installation of large and stable reflector targets in the scene would strongly support the identification errors, which assumingly both vary over time.

High-frequency sampling can be used to increase the confidence of the geomorphic change analysis. In this paper, we apply this only in the temporal domain. We will further develop our method to using spatial neighborhood information both locally and globally in the point cloud scene and improve the level of detection by leveraging spatial autocorrelation in geomorphic change.

We will continue the presented research to automatically extract dynamic areas in the target scene and subsequently characterize the morphologic change types for their spatial and temporal properties.

\section{ACKNOWLEDGEMENTS}

This work was partly funded by the Heidelberg Graduate School of Mathematical and Computational Methods for the Sciences (HGS MathComp), founded by DFG grant GSC 220 in the German Universities Excellence Initiative.

Part of the work in this article was performed within the CoastScan project and financed by the ERC-Advanced grant 291206-Nearshore Monitoring and Modeling (NEMO).

\section{REFERENCES}

AHN, 2018. Actueel Hoogtebestand Nederland. URL: http://www.ahn.nl/index.html (2019-01-11).

Avian, M., Kellerer-Pirklbauer, A., Bauer, A., 2009. LiDAR for monitoring mass movements in permafrost environments at the cirque Hinteres Langtal, Austria, between 2000 and 2008. Nat. Hazards Earth Syst. Sci., 9, pp. 1087-1094, doi.org/10.5194 /nhess-9-1087-2009.

Besl P. and McKay N., 1992. A Method for Registration of 3-D Shapes. IEEE Trans. PAMI, 14(2), pp. 239-256.

Cowell, P. J., Stive, M. J., Niedoroda, A. W., de Vriend, H. J., Swift, D. J., Kaminsky, G. M., Capobianco, M., 2003. The coastal-tract (part 1): a conceptual approach to aggregated modeling of low-order coastal change. Journal of Coastal Research, pp. 812-827.

Crown, D., W. Anderson, S., Finnegan, D., L. LeWinter, A., S. Ramsey, M., 2013. Topographic and Thermal Investigations of Active Pahoehoe Lava Flows: Implications for Planetary Volcanic Processes from Terrestrial Analogue Studies. 44th Lunar and Planetary Science Conference, p. 2.

Eitel, J.U.H., Höfle, B., Vierling, L.A., Abellán, A., Asner, G.P., Deems, J.S., Glennie, C.L., Joerg, P.C., LeWinter, A.L., Magney, T.S., Mandlburger, G., Morton, D.C., Müller, J., Vierling, K.T., 2016. Beyond 3-D: The new spectrum of lidar applications for earth and ecological sciences. Remote Sensing of Environment, 186, pp. 372-392, doi.org/10.1016/j.rse.2016.08.018.

Eltner, A., Kaiser, A., Abellan, A., Schindewolf, M., 2017. Time lapse structure-from-motion photogrammetry for continuous geomorphic monitoring. Earth Surf. Process. Landforms, 42, pp. 2240-2253, doi.org/10.1002/esp.4178.
Fey, C. and Wichmann, V., 2017. Long-range terrestrial laser scanning for geomorphological change detection in alpine terrain-handling uncertainties. Earth Surf. Process. Landforms, 42, pp. 789-802, doi.org/10.1002/esp.4022.

Hejbudzka, K., Lindenbergh, R., Soudarissanane, S., Humme, A., 2010. Influence of atmospheric conditions on the range distance and number of returned points in Leica ScanStation 2 point clouds. ISPRS Archives, 18(5), pp. 282-287.

Höfle, B., Canli, E., Schmitz, E., Crommelinck, S., Hoffmeister, D., Glade, T., 2016. 4D near real-time environmental monitoring using highly temporal LiDAR. EGU Geophysical Research Abstracts, 18(EGU2016-11295-2).

Kromer, R.A., Abellán, A., Hutchinson, D.J., Lato, M., Chanut, M.-A., Dubois, L., Jaboyedoff, M., 2017. Automated Terrestrial Laser Scanning with Near Real-Time Change Detection - Monitoring of the Séchillenne Landslide. Earth Surf. Dynam., 5, pp. 293-310, doi.org/10.5194/esurf-5-293-2017.

Kromer, R., Abellán, A., Hutchinson, D., Lato, M., Edwards, T., Jaboyedoff, M., 2015. A 4D Filtering and Calibration Technique for Small-Scale Point Cloud Change Detection with a Terrestrial Laser Scanner. Remote Sensing, 7, pp. 13029-13052, doi.org/10.3390/rs71013029.

KNMI, 2017. Metadata KNMI-stations: Hoek van Holland URL: https://projects.knmi.nl/klimatologie/metadata/hoekvan holland.html (3 Jan 2019).

Pfeifer, N., Mandlburger, G., Otepka, J., Karel, W., 2014 OPALS - A framework for Airborne Laser Scanning data analysis. Comp. Environ. Urban, 45, pp. 125-136, doi.org/10.1016/ j.compenvurbsys.2013.11.002.

Riegl LMS, 2017. Riegl VZ-2000 (datasheet). URL: https://www.3dlasermapping.com/wp-content/uploads/2017/10/ DataSheet VZ-2000 2017-06-07.pdf(11 Jan 2019).

Rijkswaterstaat, 2017. Rijkswaterstaat Waterinfo. URL: https://waterinfo.rws.nl/\#!/kaart (3 Jan 2019).

Soudarissanane, S., Lindenbergh, R., Menenti, M., Teunissen, P., 2011. Scanning geometry: Influencing factor on the quality of terrestrial laser scanning points. ISPRS J. Photogramm., 66, pp. 389-399.

Vos, S., Lindenbergh, R., de Vries, S., 2017. CoastScan: Continuous Monitoring of Coastal Change using Terrestrial Laser Scanning. Proc. of Coastal Dynamics 2017, 233, pp. 1518-1528.

Williams, J.G., Rosser, N.J., Hardy, R.J., Brain, M.J., Afana, A.A., 2018. Optimising 4-D surface change detection: an approach for capturing rockfall magnitude-frequency. Earth Surf. Dynam., 6, pp. 101-119, doi.org/10.5194/esurf-6-101-2018.

Wujanz, D., Burger, M., Mettenleiter, M., Neitzel, F., 2017. An intensity-based stochastic model for terrestrial laser scanners. ISPRS J. Photogramm., 125, pp. 146-155, doi.org/10.1016/j.isprsjprs.2016.12.006.

Wujanz, D., Avian, M., Krueger, D., Neitzel, F., 2018. Identification of stable areas in unreferenced laser scans for automated geomorphometric monitoring. Earth Surf. Dynam., 6, pp. 303-317, doi.org/10.5194/esurf-6-303-2018. 